\title{
Progress and opportunities in optical beam steering
}

Paul McManamon, Abtin Ataei

Paul F. McManamon, Abtin Ataei, "Progress and opportunities in optical beam steering," Proc. SPIE 10926, Quantum Sensing and Nano Electronics and Photonics XVI, 1092610 (29 May 2019); doi: 10.1117/12.2511987

SPIE. Event: SPIE OPTO, 2019, San Francisco, California, United States 


\title{
Progress and Opportunities in Optical Beam Steering \\ P. F. McManamon, A. Ataei \\ Exciting Technology LLC, Dayton OH, U.S.A
}

\begin{abstract}
Non-mechanical beam steering for optical systems has been important for decades, but with the dramatic increase in commercial applications, such as the driverless car, it is becoming even more important. In this paper, three decades of progress in optical beam steering is discussed, along with a comparison of approaches being developed. Non-mechanical optical beam steering is compared against mechanical steering approaches. Unique space fed beam steering approaches are discussed using different active electro optical media. Some electro optical beam steering systems are explained in detail and some methods to improve the efficiency are presented.
\end{abstract}

\section{Summary of Existing Mechanical Beam Steering Systems}

Mechanical approaches are being used now for most applications, so non-mechanical approaches will need to displace existing mechanical approaches in various applications. The main mechanical beam steering approaches are; Gimbals, fast steering mirrors (FSMs), Risley prisms, rotating polygons, and two micro-mechanical approaches, lenslet arrays and MEMs. 
Often the last two are grouped with non-mechanical approaches, because the mechanical motion is small.

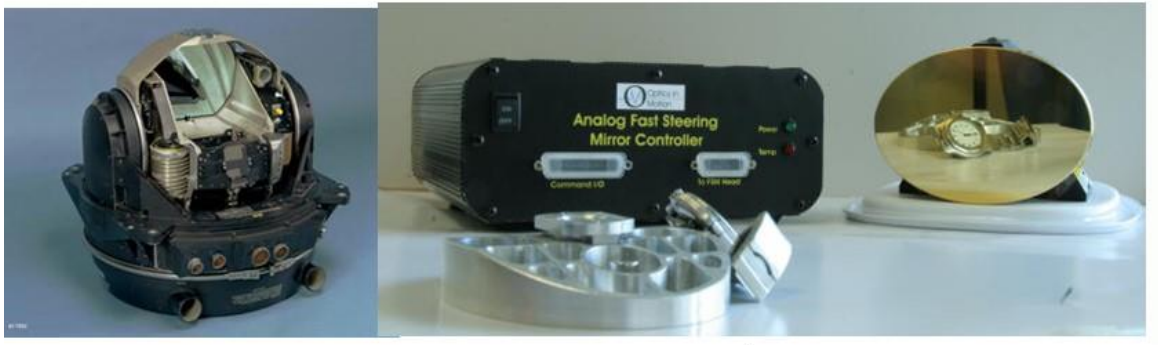

a

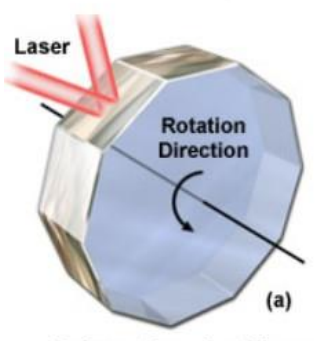

Polygon Scanning Mirror

d b

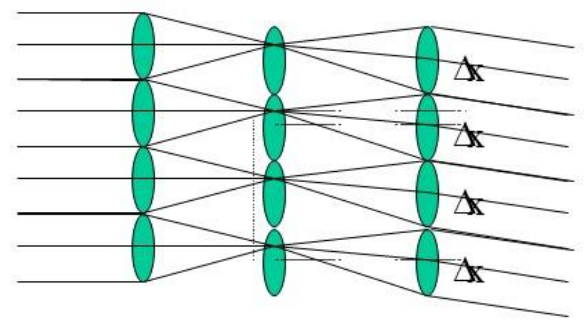

$\mathrm{e}$

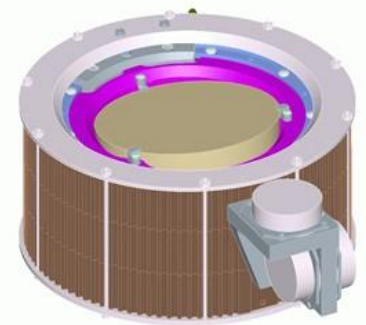

C

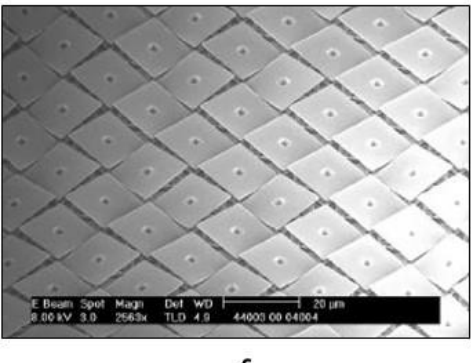

f

Figure 1 : Mechanical Approaches to Beam Steering, a) Gimbal, b) Fast Steering Mirror (FSM), c) Risley prism, d) Rotating Polygon, e) Lenslet Array, and d) MEMs array.

Gimbals are the go to devices for high performance EO systems. The military uses them, and most long-distance commercial sensors, such as people watching animals in the wild from a long distance. Fast Steering Mirrors, FSMs, are often the mechanical device that nonmechanical steering systems must beat for short range, or low cost, applications. They are moderately fast, reliable, and cheap. Their big issue is they are small and therefore the beam should be magnified after steering. The steering angle is decreased linearly with magnification, so a large steering angle with a small beam becomes a small steering angle with a large beam. While FSMs can move fast, there is a settle time of $6 \mathrm{msec}$ or more for accurate pointing. Risley prisms and polygons both benefit from simple rotation as the form of motion, instead of more complicated forms of motion. Grocery store scanners use rotating polygons. For moderate size beams Risley prisms are very interesting. Risley gratings, which are lighter, are also used, although they have dispersion. Risley gratings are appropriate for narrow band systems but not broadband. Lastly, we will mention lenslet arrays and MEMS. Both use micro-motion, so these devices can be classed with non-mechanical steering devices. MEMs have significant interest currently. They can have issues with pointing stability in dynamic environments, but they can steer moderately fast, and can be manufactured inexpensively. Another issue with MEM is if the MEMs array is phased up. To phase up a MEMs arrays, and obtain the diffraction limit of the array size, requires both tip and tilt, and piston motion of the array ${ }^{1,2}$, unless the piston motion is obtained by another device. Lenslet arrays can steer to large angles with small motion, and can be made large, but have not caught on for many applications ${ }^{3}$. They require 
three lens layers. The middle lens is used to make sure the final lens captures all the light being steered.

\section{Non-Mechanical Approaches to Optical Beam Steering}

To date most optical phased array, OPA, steering uses space feed phased array steering, as compared to individual transmit/receive module based steering such is often used with phased array microwave radars, and to date most OPA steering is one dimension at a time. Traditional radar phased arrays have individual phase elements that are at half wavelength spacing, or smaller. If we consider an $\mathrm{X}$ band radar at $10 \mathrm{Ghz}$ the wavelength is $3 \mathrm{~cm}$. Wavelength spacing for individual phase shifters with a $3 \mathrm{~cm}$ wavelength would mean the pitch between individual phased array elements is no larger than $1.5 \mathrm{~cm}$, half wavelength spacing. It is possible to build, and address, individual RF transmit/receive (T/R) modules that are $1.5 \mathrm{~cm} \times 1.5 \mathrm{~cm}$ on the radiating/receiving surface. If we want a square radar aperture that is $75 \mathrm{~cm} \times 75$ we will need a $50 \times 50$ array of $\mathrm{T} / \mathrm{R}$ modules that are $1.5 \mathrm{~cm}$ on a side, or 2500 individual elements. If we make this array round, instead of square, the number of elements decrease to about 2000 elements. Now let's consider a similar optical phased array based on T/R modules. We will assume an optical wavelength of $1.5 \mu \mathrm{m}$. If we use a half wavelength pitch that means we will have a $.75 \mu \mathrm{m}$ between elements. Optical apertures usually are not as large as microwave apertures, so let's assume our optical aperture is only $30 \mathrm{~cm}$ x $30 \mathrm{~cm}$. To build this aperture we will need to have 400,000 elements on a side, and a total of 160,000,000,000 T/R modules in a square aperture, or about $1,250,000,000,000$ elements in a round aperture that is $30 \mathrm{~cm}$ in diameter. This same array would have 400,000 elements in one direction. If we use two crossed one dimensional arrays we would need 800,000 elements. This is still a large number. To put these numbers in context however the iPhone 6 in 2015 had 2,000,000,000 transistors on a

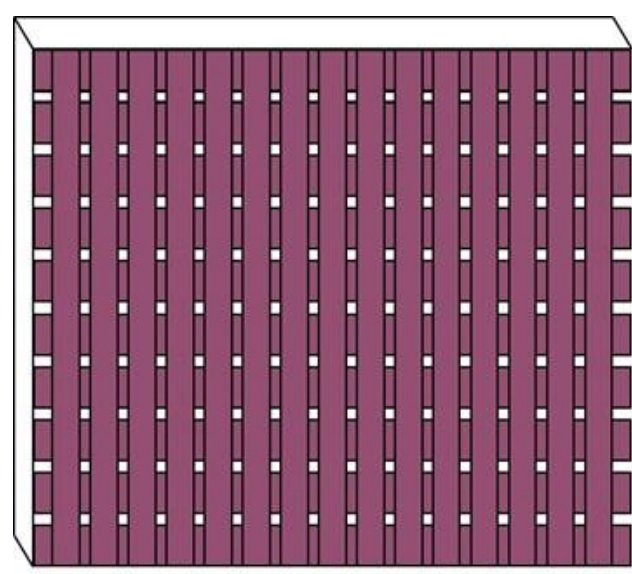

Figure 2: Two crossed one dimensional space fed phased arrays

chip, whereas a Pentium 4 in 2001 had 2,000,000 transistors. ${ }^{4}$ This is a factor of 1000 increase in 14 years. We do see rapid progress in addressing larger and larger arrays of transistors on a chip. Also, for a 2D array each T/R module has to be $.75 \mu \mathrm{m} \times .75 \mu \mathrm{m}$ on the surface of the apertures, which is very small. In today's technology this is impractical. As a result, the optical community has started working optical phased arrays that are one dimensional at a time and are space feed. A space feed array changes the phase of an optical beam passing through it, resulting in steering the optical beam. It could be possible in the relatively near term to do T/R modules in one direction, while using a different non-mechanical approach in the other direction. Figure 2 shows two crossed one dimensional space fed phased arrays.

There are two fundamentally different ways of steering an optical beam with no moving parts. The most common way to build OPAs, is by dynamically creating an optical path difference, OPD. This OPD is equivalent to a certain phase difference at a particular wavelength. A second approach is to create a phase difference, which is equivalent to a certain OPD at a given 
wavelength. Most OPA approaches are OPD based approaches. The phase based approach is based on the work of Pancharatnam in his classic 1955 paper. $^{5}$ We will discuss both fundamental approaches, as we discuss specific OPAs that use one, or the other basic beam steering approach.

\section{1: Modulo 2 $\pi$ Thin Space Fed Beam Steering Approaches \\ 2.1.1: Modulo 2 $\pi$ Architecture}

For a narrow wavelength it is possible to take advantage of the fact that light is a sine wave. With sine waves it does not matter if we have $0,2 \pi, 4 \pi$ or $2 n \pi$ phase shift. In other words, the OPDs of any integer multiplied by wavelength $(n \lambda)$ would be the same from a phase point of view. Therefore, as one moves across the width of the prism, one can subtract $2 \pi$ n of phase every time the phase reaches $2 \pi \mathrm{n}$, resulting in a saw tooth phase profile. The unfolded phase,

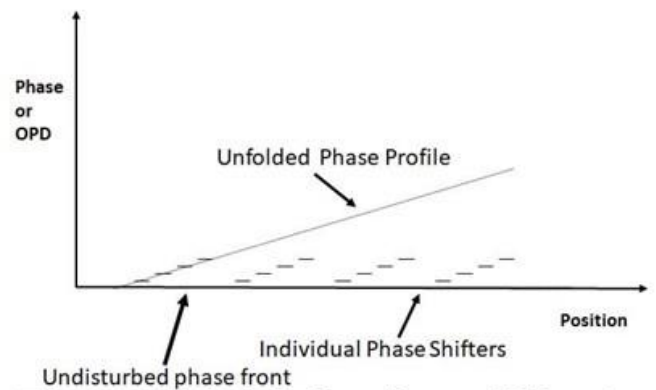

Figure 3: Modulo $2 \pi$ phase shifting to create beam steering which is called a modulo $2 \pi$ phase profile, looks like the phase profile that would result from propagation through a full prism, and steers light in the same manner. Because a reset occurs every time the Optical Path Delay, OPD, reaches $2 \pi \mathrm{n}$, smaller angles have a larger reset period. As seen in Figure 3, when the OPD reaches a multiple of one wavelength $(\lambda)$, a reset occurs because an OPD of zero and a multiple of one wavelength would be the same from a phase point of view. It should be noted that resets can be initiated at OPDs that are not a multiple of one wavelength. For example, the reset can occur after reaching an OPD of $1.1 \lambda$. The benefit of using a modulo $2 \pi$ phase profile is that the required OPD can be small. The maximum required OPD is approximately equal to the wavelength of the light being used. The modulo $2 \pi$ steering approach makes the beam steerer very wavelengthdependent (dispersive) $)^{6,7}$. The largest angle one can steer to using the modulo $2 \pi$ approach is determined by the size of the smallest reset possible for a desired steering efficiency.

If one illuminates the full array of phase shifters with a Gaussian beam, then any individual phase shifter will have an approximately uniform irradiance distribution across it. For a circular aperture, uniformly illuminated, the full width beam divergence at the half power point is ${ }^{8}$ :

$$
\theta \cong \frac{1.03 \lambda}{d}
$$

where $\theta$ is beam divergence, $\lambda$ is wavelength of the electromagnetic radiation, and $d$ is the width of the individual radiator. If phase can be locked among many individual radiators, the beam will become narrower in angle proportional to the increase in the effective size of the radiator. If the full array is uniformly illuminated then we can substitute:

$$
D=n d
$$

In Eq.(2), where $n$ is the number of individual radiators assembled to make the large radiator, and where we have assumed that the pitch of the radiator separations is equal to the width of the radiator (i.e. unity fill factor). For Gaussian illumination of the full array the effective size of the large aperture is reduced and the beam divergence increases. The allowed amount of 
clipping of the Gaussian beam by the aperture array determines how much the effective aperture size is reduced. By adjusting the phasing among the individual elements, the narrow beam can be steered under the envelope of the larger beam resulting from an individual radiator. Phased array microwave radars steer the beam to angles larger than 45 degrees. To do this, the radars use individual radiators that are at a half wavelength spacing or closer. In radar the conventional discussion of half wavelength spacing says individual phase adjustable radiators must be no larger than half wavelength to reduce grating lobes ${ }^{9}$. This is a different view of the same physics. From Eq. (1) if $d$ equals one half of $\lambda$ then $\theta=2.06$ radians, or 118 degrees. This is the full beam width at the half power points. We could steer plus or minus 45 degrees and still be above the half power point, neglecting the cosine factor loss in the projected area of the aperture.

One of the main efficiency considerations is the fly back region, which is determined by fringing fields ${ }^{10}$. This effect is a result of the inability of the device to change its electric field profile instantaneously in space. The fly back region essentially reduces the fill factor of the grating. The fly back region depends primarily on the structure of the modulo $2 \pi$ beam steering device. Some active EO materials, such as liquid crystals, also have limitations on quickly the index can change in space, which can act similar to fringing fields in limiting steering efficiency, causing a region that steers the wrong direction.

It should be noted that the size of the reset period $(\Lambda)$ will determine the deflection angle as follow;

$$
\tan \theta=\frac{\lambda}{\Lambda}
$$

Where the reset period, $\Lambda$, consists of q electrodes of size w, each separated by the spacing of s. Therefore, the deflection angle will depend on the number of electrodes in each reset, the size of each electrode and the spacing between the electrodes as follow;

$$
\tan \theta=\frac{\lambda}{q(w+s)}
$$

The active layer thickness should be made as thin as possible to minimize the fly back region. The electrode size, spacing and the number of electrodes in each reset should be optimized to not only to satisfy the desired deflection angle, (Eq. (4)) but also to maximize the efficiency. The number of electrodes in each reset also imposes another effect called, quantization effect, which will be explained later in this paper.

Eq. (5) gives the efficiency of liquid crystal based beam scanner due to fly back region effects.

$$
\eta=\left(1-\frac{\Lambda_{F}}{\Lambda}\right)^{2}
$$


Where $\eta$ is efficiency, $\Lambda_{F}$ is the width of the fly back region, and $\Lambda$ is the width between

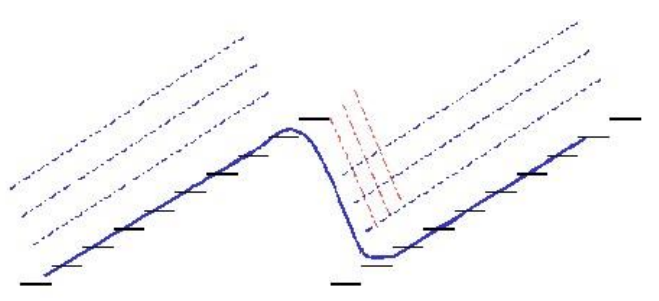

Figure 4: The effect of fringing fields on phase profile resets $^{11}$. Figure 4 shows that during the fly back portion of the phase profile the beam is deflected in to the wrong direction. Fringing fields make it impossible to impose an electric field that stays only between the small electrodes. Instead the field expands outward to each side of the small electrode. As a rule of thumb, the narrowest width of a voltage region above an electrode is about the thickness of the layer between the electrode and the ground plane ${ }^{12}$. Since the thickness of the active media layer is often larger than the spacing between the electrodes, it can be seen that fringing fields can cause a significant loss in efficiency. The OPD in the liquid crystal based scanners is also a faction of the active layer thickness. For transmissive beam steering the cell has to be about as thick as required to obtain one wavelength, or $2 \pi$ phase, of OPD. With a birefringence of .3 this means the cell has to be about 3.3 times one wavelength in thickness. According to Eq. (4) and Eq. (5) which gave the deflection angle and efficiency, it can be shown how fast the efficiency drops by deflection angle for a conventional liquid crystal based thin film scanner

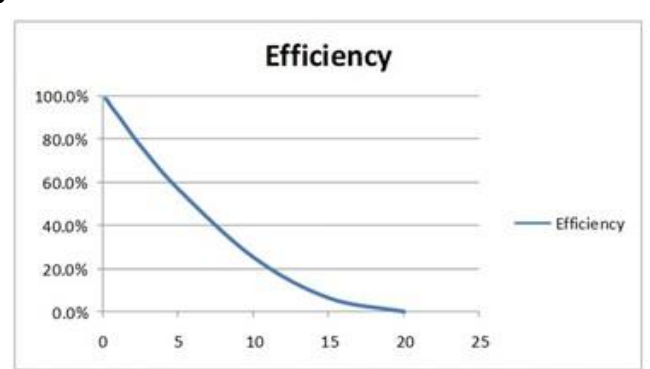

Figure 5: Efficiency vs. deflection angle limited by fringing field effects

for a conventional liquid crystal based beam steering (Figure 5). Therefore, if we want high efficiency, we need to limit the steering angles used for saw tooth phase profile liquid crystal continuous steering to very small angles. As seen in Figure 5, for a quarter of a degree angular steering we only achieve about $98 \%$ steering efficiency. For 1 degree steering we are down to $90 \%$ efficiency. If we need 1 degree steering in both azimuth and elevation we need to square that loss. This is even a major limitation for use as the fine angle steering before a wide-angle course steering element.

The second contribution to steering efficiency is from the discrete nature of the phase steps. Eq.6 gives the loss in efficiency from using discrete steps.

\begin{tabular}{c}
\multicolumn{2}{c}{ Efficiency vs. \# of steps } \\
$\begin{array}{cc}\mathrm{q} & \text { Efficiency } \\
8 & 95.0 \% \\
10 & 96.8 \% \\
12 & 97.7 \% \\
16 & 98.7 \% \\
20 & 99.2 \%\end{array}$
\end{tabular}

Table 1: Steering Efficiency Limited by quantum effect

$$
\eta=\left(\frac{\sin (\pi / q)}{\pi / q}\right)^{2}
$$

Where $\eta$ is efficiency and $q$ is the number of steps in a ramp (reset period). Table 1 shows the efficiency vs. number of steps in a ramp. Some early liquid crystal beam steering work avoided the loss associated with discrete steps, discussed above ${ }^{13}$. In this approach a linear ramp in electric field was used instead of discrete phase steps. Initially it was not obvious which approach would be preferred, but the discrete steps did not provide a significant loss, and the linear region of the voltage vs. phase shift was only a small portion of the full phase shift available from a liquid crystal cell, so it was necessary to make the LC cell thicker. The major loss using liquid crystal beam deflection had to do with 
fringing fields. This loss was not affected by using discrete steps vs. a linear profile. The net result is the linear liquid crystal phase ramp approach to steering optical beams was not pursued further at that time. If a different active material was used this approach could be attractive. Fringing fields are the main issue to address to increase efficiency of modulo $2 \pi$ beam steering. This is being addressed and can result in steering efficiently to larger angles ${ }^{14}$.

\subsection{2: Liquid Crystal Modulo 2 $\pi$ OPAs}

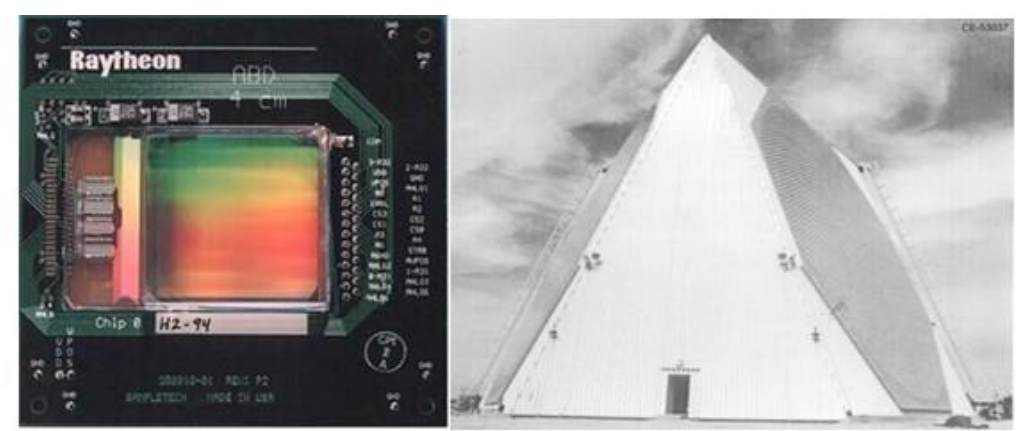

Figure 6 : (a, on left) Early Raytheon OPA with 40,000 phase shifters on 1 micron pitch, (b, on right) Pave Paws Search Radar

The initial OPA work we did in the $80^{\prime} \mathrm{s}$ was modulo $2 \pi$ beam steering using liquid crystals. Initially we did not think about fringing field effects, so Raytheon made a 4 $\mathrm{cm}$ wide OPA on $1 \mu \mathrm{m}$ pitch, shown in Figure 6a. In contrast the microwave Pave Paws radar, shown in Figure $6 \mathrm{~b}$, has 7,000 phase shifters. This gives an idea of the difference in scale of RF vs optical phased arrays. Since the frequency of Pave Paws search radar is low, its phase shifter are significantly larger than an $\mathrm{X}$ band radar. The AN/FPS- 115 radar consists of two phased arrays of antenna elements mounted on two sloping sides of the $105 \mathrm{ft}$. high transmitter building, which are oriented $120^{\circ}$ apart in azimuths. The radar operates in the UHF band, between 420 $450 \mathrm{MHz}$, has a wavelength between $71-67 \mathrm{~cm}^{15}$.

In the 90's a small business, Boulder Nonlinear Systems, BNS, started to also make LC OPAs. The 1st commercial OPA was by BNS in 1999, shown in Figure 7a. It is a $1 \times 4096$ OPA, on 1.8 micron pixel pitch. The device is $.74 \mathrm{~cm} \mathrm{x} .74 \mathrm{~cm}$ and was the

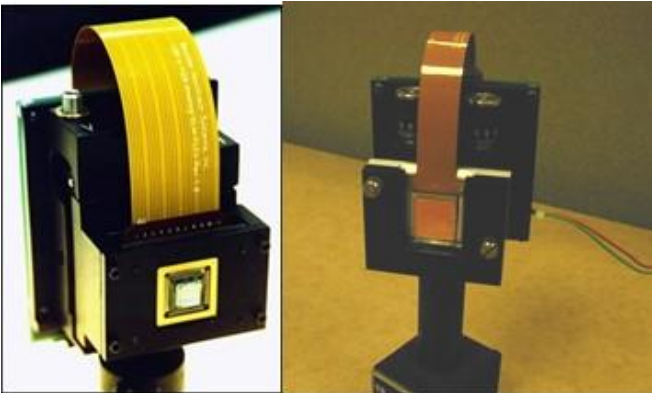

Figure 7 : (a, on the left), is the $1 x$ 4096 OPA (1999), and (b, on the right), is the $1 \times 12228$ array (2003) winner of Circle of Excellence Award in 2000. It was developed under an SBIR contract to the organization Dr. McManamon was in at the time, AFRL/SN. Then BNS developed a larger OPA, which became available in 2003 . It is $1 \mathrm{x} 12,228$ on a 1.6 micron pitch. It took up to 13.2 volts to address it, and was 19.2 by $19.2 \mathrm{~mm}$ in size. One of the reasons we started with liquid crystals is the large birefringence possible, and the low voltage required to impose that birefringence.

\subsection{3: EO Crystal Modulo 2 $\pi$ OPAs}

A thin, modulo $2 \pi$, beam scanner consists of an optically active layer sandwiched between electrodes. Voltages are applied to change the refractive index of the active layer to delay the incident light for at least one wavelength. Any type of active materials such as Liquid crystals, Electro Optical (EO) crystals, or quantum dot material can be used as the active layer. Figure 
8 shows the performance of a particular KTN based modulo $2 \pi$ scanner before and after applying a voltage. In this case the beam is steered 5 degrees. With conventional addressing approaches steering angles this larger cannot be obtained.

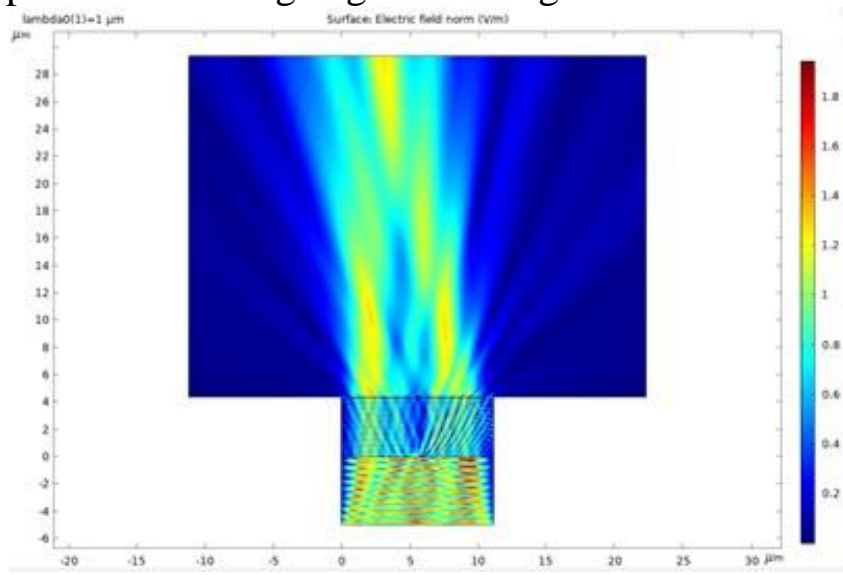

(a)

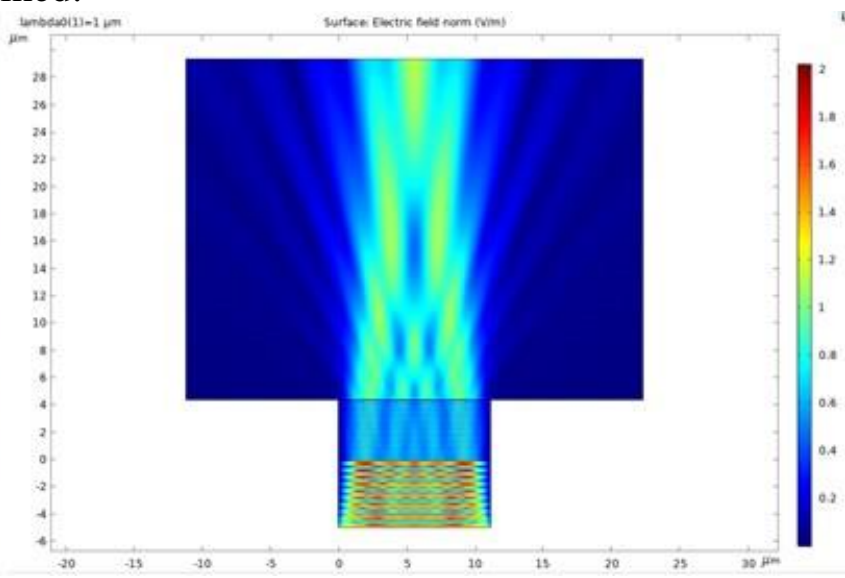

(b)

Figure 8, the performance of a KTN based thin film scanner, a) before applying the voltage, b) after applying the voltage

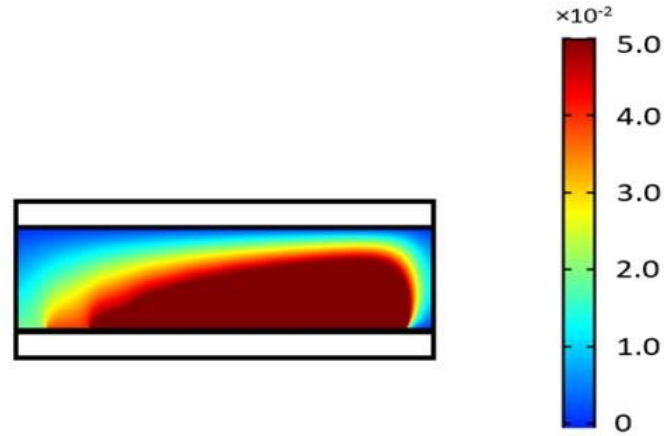

Figure 9: Index variation within a unit cell. The electrode is resistive with two different voltages applied. An optical beam propagating through this unit cell will acquire a full wave of $O P D$.

smaller for wider angles. As mentioned, the structure of the thin film beam scanner may affect the fly back region. As seen in Figure 10, when the size of the electrodes (w) is almost the same as the active layer thickness (1), the fly back region will be smaller and hence the efficiency will be higher. The electric field generated by the applied voltage on each electrode will also be affected by the next electrode. Therefore, in addition to the size of the electrodes (w) and the thickness of the active layer (l), the spacing between the electrodes (s) will affect the fly back region and hence the efficiency of the scanner.
Figure 9 shows the voltage profile for 3 um thick KTN crystal. Voltage from 0 to $V_{\lambda} / 2$ is applied on the first half electrodes of the reset period, then the voltage is reset to $-\mathrm{V}_{\lambda} / 2$ on the next electrode. For the second half electrodes, the applied voltages are gradually increased to zero and the pattern is continued for the next reset period. That reset period is larger for smaller deflection angles and is

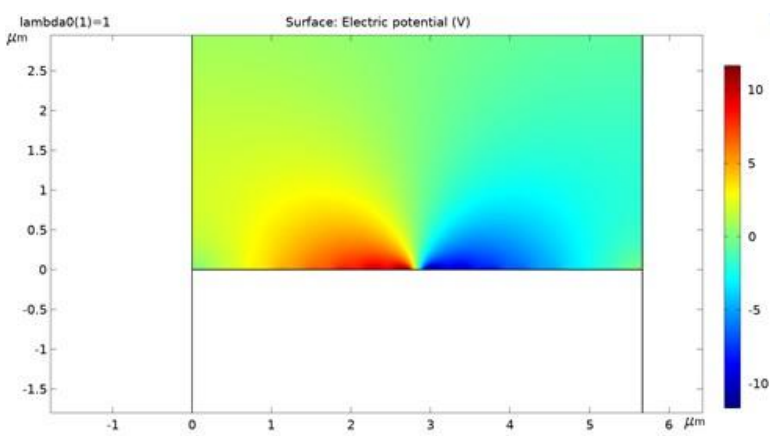

Figure 10; Voltage profile applied on 3um thick KTN crystal 


\subsection{4: Quantum Dot Modulo 2 $\pi$ OPAs}

Another active medium that can be considered is quantum dots. ${ }^{16,17}$ We can use any active medium whose index of refraction changes based on applying a voltage. Quantum dots have the advantage that both polarizations can be changed based on applying voltage rather than just one polarization. Not much has however been published using quantum dots for index change.

\section{2: True Time Delay EO Crystal Steering Approaches}

2.2.1: SEEOR This device uses liquid crystals as an active cladding layer in a waveguide architecture where light is confined to a high index core and the evanescent field extends into

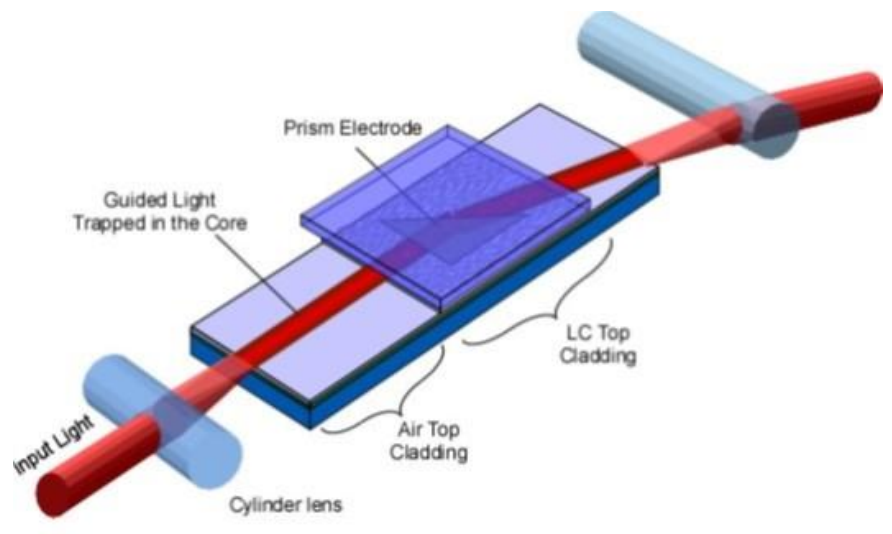

Figure 11: SEEROR beam steering basic design the variable-index liquid-crystal cladding. This allows very large optical path delays, about $2 \mathrm{~mm}$, so for small apertures it eliminates the need for a $2 \pi$ resets. Because of the long path this approach can be a true time delay OPA. There is therefore no fringing field issue, because there are no resets. Also, because the liquid crystal layer is thin these devices can be relatively fast, under $500 \mu \mathrm{sec}$ in response time. In plane steering is accomplished by changing voltage on one or more prisms filled with liquid crystals, as shown in Figure 11. Out of plane beam steering is based on the waveguide coupler designed by R. Ulrich at Bell Labs in 1971. This is shown in Figure 12. In any waveguide, if the cladding is too thin light will leak out of the guided mode. In a planar slab waveguide Snell's law gives the propagation angle of the escaping light, since it is possible to tune the effective index of the waveguide it is

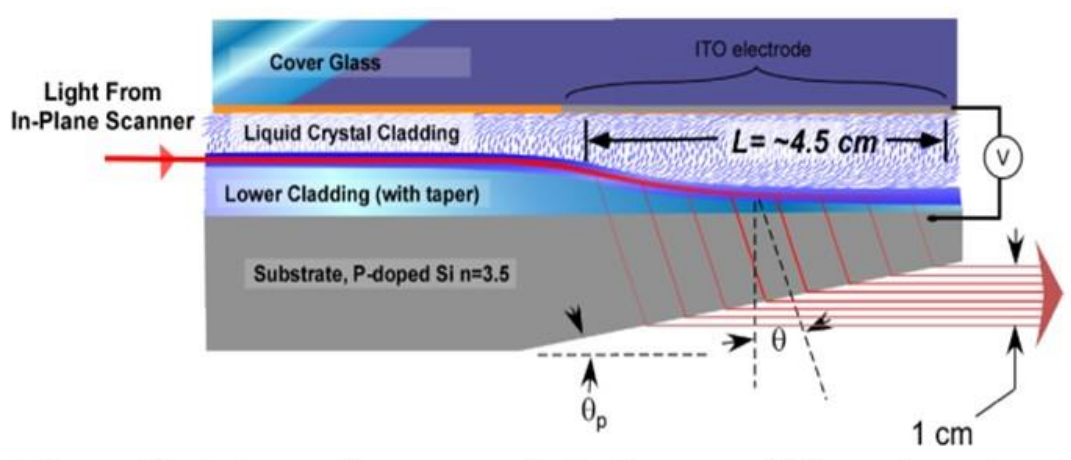

Figure 12: Out coupling approach for the second dimension using SEEOR beam steering therefore possible to tune the angle of the escaping light. This waveguide based liquid crystal based beam steering can steer in one direction over wide angles, such as 40 degrees, rapidly. In the grating out coupled dimension the steering angle is more limited, to maybe 15 degrees in either direction. This is the second steering dimension. One main limitation on this technique is the size of the apertures, which will be limited to a little, under $1 \mathrm{~cm}$ on a side or less. Currently the loss using this technique is fairly high, on the order of $50 \%{ }^{18}$. 


\subsection{2: Bulk EO crystal Beam Steering}

A key constraint on bulk EO crystals will be crystal thickness, which is proportional to the required voltage, as discussed above. Anticipate kilovolt voltage ranges for bulk EO crystal based steering.

LiNiobate has been used a long time for steering beams, but it has a low EO coeficient, so it requires a high voltage. More recently high EO coefficient materials, such as $\mathrm{KTN}, \mathrm{BaTiO}_{2}$, PZT, SBN, and PMN-PT have been investigated because of higher EO coeficients, and the potential for lower required voltage. A critical issue for bulk beam steering is the required voltage. There is a trade that as the steered beam gets larger, so does the voltage, but small beams require magnification, which reduces steering angle. Also, beam walk off results from deflecting the beam inside the crystal, so a portion of the beam might hit the side of the crystal. This requires a larger crystal increasing required voltage. Beam walk off is more of an issue for the first dimension steered, because the 1st dimension has already been steered, and will have a more significant walk off problem in the second crystal. If we have a $20 \mathrm{~mm}$ long crystal to steer each dimension then by the end of the second crystal we should have about an effective $30 \mathrm{~mm}$ walk off length. In the first $20 \mathrm{~mm}$ long crystal we should only have about an effective $10 \mathrm{~mm}$ walk off length because the steering occurs gradually over the length of the crystal so initially the beam is not steered to the final steering angle within the crystal. The angle we obtain in the first crystal however continues on into the second crystal. The steering has already occurred when light hits the crystal that steers the second direction. In the dimension steered by the first crystal the second crystal will have to be wider to avoid the beam hitting the side wall due to beam walk off.

It is interesting that the OPD required to steer to a certain angle/aperture product remains the same for a given crystal regardless of the width of the crystal we start with. We can think of an EO crystal as a capacitor. The capacitance of an EO crystal with length of 1 , width of $w$ and height of $d$ is given by:

$$
C=\frac{\varepsilon_{r_{i j}} \varepsilon_{0} L w}{d}
$$

The dielectric constant in Eq. (7) can be different for different crystal orientations, and for different crystals. We see that to keep the capacitance low, we would like a low dielectric constant, but we will learn a high dielectric constant can allow a larger steering angle per applied voltage which may be desirable. Steering to a given angle requires a certain voltage across a crystal.

One way to steer to a certain angle, using a prism type OPD profile, will be to create an Optical Path Difference, OPD, on one side of the crystal, preferably with a linear prism profile in OPD across the crystal. One side of the crystal will have a maximum OPD, and the other side will have zero OPD. This change in OPD across a crystal creates a tilt to the outgoing wave front. The change in OPD is given by:

$$
O P D=\triangle n L
$$

where $\mathrm{L}$ is the length of the crystal, or of the area of the crystal with a changed index of refraction, and $\Delta \mathrm{n}$ is the change in index of refraction. While we can develop a larger OPD by using a longer interaction length, up to the size of the largest available crystal, it is desirable to create a larger $\Delta \mathrm{n}$ instead of a larger interaction length as the method of developing a given OPD. As mentioned earlier a larger interaction length not only makes the beam steerer larger, but creates more of a walk off issue, where a portion of the beam could hit the side wall of the 
crystal unless the beam is kept very small compared to the crystal size. The amount of OPD generated determines the angle/aperture product for beam steering. A wider aperture means steering to a smaller angle, but does not change the angle/aperture product.

Because of the higher index of refraction, we will not need to steer to as large an angle inside the crystal to generate this angle upon leaving the crystal. We can use Snell's law at small angles to determine what angle we have to steer to.

$$
\frac{\vartheta_{0}}{\vartheta_{f}} \approx \frac{n_{f}}{n_{0}}
$$

Where $\mathrm{n}_{0}$ is the index in air and $\mathrm{n}_{\mathrm{f}}$ is the index of refraction in the crystal. Solving for $\vartheta_{f}$ we have:

$$
\vartheta_{f} \approx \frac{n_{f}}{n_{0} \vartheta_{0}}
$$

The index of refraction in air is 1 . The approximate index of refraction for KTN is 2.29 , for PMN-PT is 2.47, and for SBN is 2.35. Using these values reduces the required internal steering angle for prism type steering.

We can generate a $\Delta \mathrm{n}$ either by a linear electric field or by a quadratic electric field, depending if the crystal has a large linear EO effect or a large Kerr effect. KTN has a large Kerr effect, with the Kerr effect as the best EO coefficient to use to create a change in index, so uses a quadratic function of the electric field to create a change in the index of refraction. PMN-PT has also a large Kerr effect, although could be used in a linear mode as well. $\mathrm{SBN}$ and $\mathrm{BaTiO}_{3}$ are linear materials with electric field, so use an EO, or Pockels, effect. EO, or Pockels effect, crystals need to be poled, but Kerr effect crystals do not need to be poled. The linear EO effect creates a $\Delta \mathrm{n}$ using;

$$
\Delta n=-\frac{1}{2} n^{3} r_{i j} E_{j},
$$

$\mathrm{E}_{\mathrm{j}}$ is just voltage divided by crystal thickness in an appropriate direction.

Reference $3^{19}$ shows the Kerr effect equation as being:

$$
\Delta n=-\frac{1}{2} n^{3} S_{i j} E_{j}^{2}
$$

Which is similar to the linear Pockels cell effect, except for the square electric term.

\section{3: Steering Using Gratings}

2.3.1: Wavelength Change

The grating equation is given by:

$$
\sin (\theta)=\frac{n \lambda}{d}
$$

And for small angles the steering angle is proportional to wavelength. Regardless if the steering angle is exactly proportional to wavelength or not you can see that if we have a grating we can change the steering angle by changing wavelength. Therefore IF we have an EO system capable of changing wavelength we can do non-mechanical beam steering.

\subsection{2: Volume Holographic Gratings}

Volume (thick) holograms offer the potential to implement large angle steering with high efficiency $^{20}$. Once the hologram is developed it will diffract an incident signal beam into the direction of the reference beam, thereby steering the signal beam, while being transparent to beams coming it at different angles. Through the use of multiple holograms, multiple 
discrete steering angles can be addressed. The number of steered angles increases linearly with the number of holographic gratings. This is the reason holographic beam steering has lost its popularity compared to polarization birefringent gratings. In the 90's the main wide angle beam steering approach was holographic gratings, made by Leon Glebov, at CREOL, and the company he started called Optigrate, using high fidelity, rugged, medium for writing holograms is photo-thermal glass ${ }^{21}$. The steering angles can be large, allowing us to achieve the large Lagrange Invariants that we desire. Each glass holographic grating can have $>99 \%$ efficiency ${ }^{22}$. When two holograms are written in a single piece of glass the efficiency can still be over $98 \%{ }^{23}$.

Many layers of holographic glass can be placed back to back with low loss. For example, we could use eight holograms in each direction, azimuth and elevation. If each hologram steers

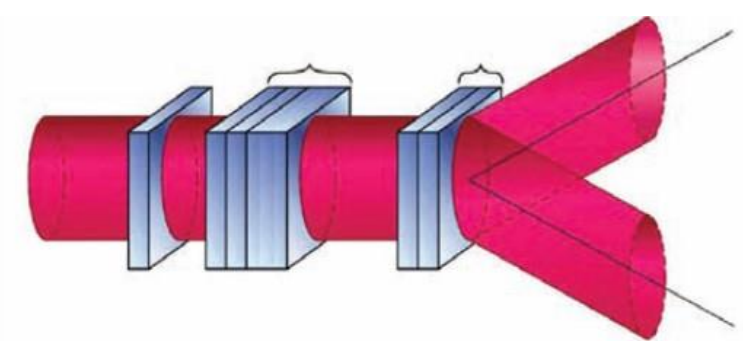

Figure 13 : Wide angle beam steering using Holographic Glass to an angle separated by five degrees from the adjacent angle, then we have a total field of regard of 40 degrees, broken up into eight zones of five degrees each. The incoming light is only diffracted by the holographic grating that has light input at the proper angle, and wavelength. There is no additional diffractive loss by using more gratings. More volume holograms do however introduce reflection, scattering, and absorption losses. In addition the thickness of the grating stack increases. Only one grating in each dimension is set to steer light input at a given angle, dividing the steering into zones. Steering inside of each zone is referred to as filling each zone. This requires the use of a second beam steerer in each dimension after the stack of volume holograms. This wide angle steering approach therefore requires two moderate angle, continuous, beam steering devices, one before (for zone selection) and one after (for zone fill) the stack of volume holograms. This steering approach has demonstrated continuous beam steering over a field of regard greater than 45 degrees $^{24,25}$. It would be possible to use this technique to steer both polarizations of light, but it would mean doubling the number of beam steering elements. If we want an 80 degree field of regard and 5 degrees per step, then we would need to have 16 gratings in each dimension. Even at $.5 \%$ loss per grating that would mean a $16 \%$ loss when both dimensions are included. It is critical to reduce losses per stage to a minimum if large angle deflection is desired at high efficiency. A single piece of holographic glass is about $2 \mathrm{~mm}$ thick. This can cause limited walk off issues in a large stack, due to $4 \mathrm{~cm}$ thickness for 20 gratings. By walk off we mean the beam can move off the active area of the substrate or can hit the side wall because it is essentially steered inside of a tunnel.

While this is a very effect method of wide angle beam steering it has been abandoned once polarization birefringent gratings, to be discussed later, became available. 


\section{4: Chip Scale Transmit/Receive Module Based OPAs}

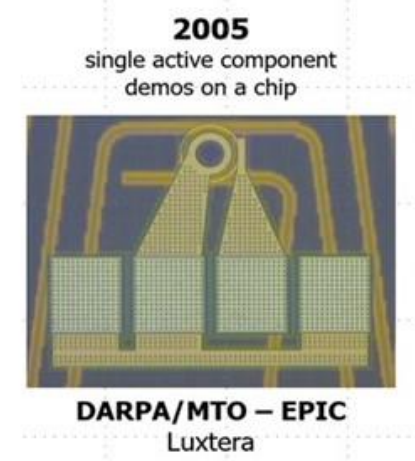

2015

"LIDAR-on-a-chip" with 500 components

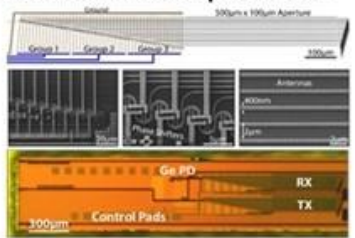

DARPA/MTO SWEEPER/E-PHI

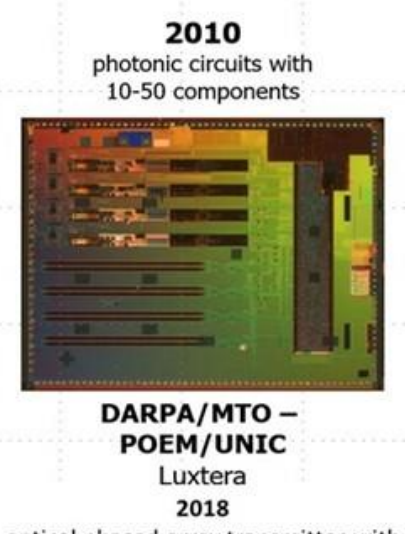

optical phased array transmitter with $>3,000$ components

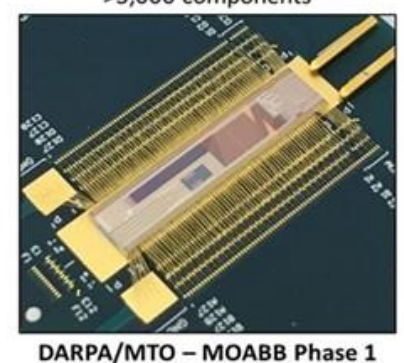

Chip scale OPAs are also being pursued $^{26}$. DARPA had a program called Sweeper, which jump started this approach, and resulted in some interesting papers, but not very practical devices. The devices were small, and not do both transmit and receive. More recently there is a DARPA program called MOABB that is attempting to make chip scale devices larger, and to allow both transmit and receive. The basic physics of optical phased arrays are the same whether one uses a chip to create OPD or uses birefringent liquid crystals. The chip approach may not however have fringing field issues. The approach shown in the reference 24 does create OPD, and does use modulo $2 \pi$ beam steering. This particular chip is 64

Figure 14: Progression of DARPA Chip Scale programs

x 64, with a pitch of $9 \mu \mathrm{m} \times 9 \mu \mathrm{m}$, making the array $\sim 576 \times 576 \mu \mathrm{m}$. The individual antenna radiators inside a pixel are $3.0 \mu \mathrm{m}$ in length, $2.8 \mu \mathrm{m}$ in width. A second early chip scale optical phased array ${ }^{27}$ uses random element pitch to reduce side lobes, but is also a very small chip. Often

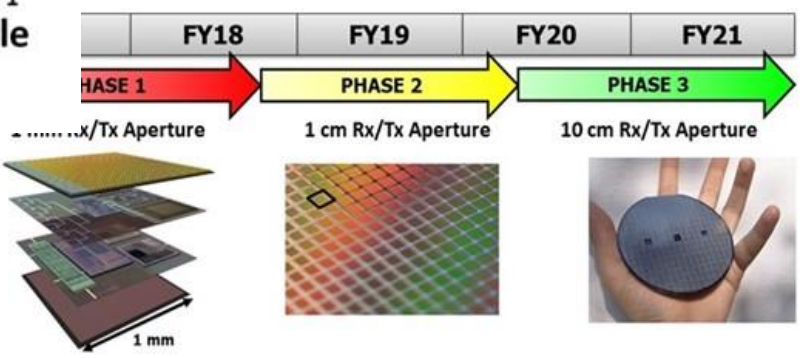

Figure 15: MOABB Phases when phased array individual elements are larger than a wavelength people talk about the spurious side lobes. Another way to consider this is that the largest full width, half maximum angle that can be steered to without significant side lobes is limited by the size of the individual phase shifters, as given in equation 1. Since steering is only in one direction, and a higher efficiency than 50 percent (half max) is desired, it is necessary to restrict the steering angle to about one-fourth of that value. If $3 \mu \mathrm{m}$ is used as the size of the radiator, and $1.5 \mu \mathrm{m}$ is the wavelength, steering can be done to about $1 / 8^{\text {th }}$ radian, or about 7 degrees. In one example, ${ }^{28}$ the chip is only about $576 \mu \mathrm{m}$ - a very small aperture. Assuming a $10 \mathrm{~cm}$ beam, this implies a magnification of about a factor of 174, reducing the 7 degree steering angle to .04 degrees.

Over time the steering arrays will become larger, and the individual steering elements will became smaller. This article just discusses transmitting, not receiving, which is another required growth area. It is likely that nano fabricated phased arrays will become an interesting option for steering to small angles, using magnification after steering.

More recent progress in chip scale OPA is discussed in reference 27. ${ }^{29}$ Lockheed Martin was one of the MOABB contractors, with support from the University of California. 
They have gotten the spacing down to 1.3 microns, allowing steering up to plus or minus 10 degrees. Reference $28,{ }^{30}$ by the DARPA program manager, Gordon Keeler, shows progress in increasing chip count. Phase 2 and 3 of MOABB will progress the chip count higher, as can be seen from Figure. Columbia University and Analog Photonics are two phase 2 MOABB contractors.

It is interesting to extrapolate from figure 14, where it states the Sweeper program had

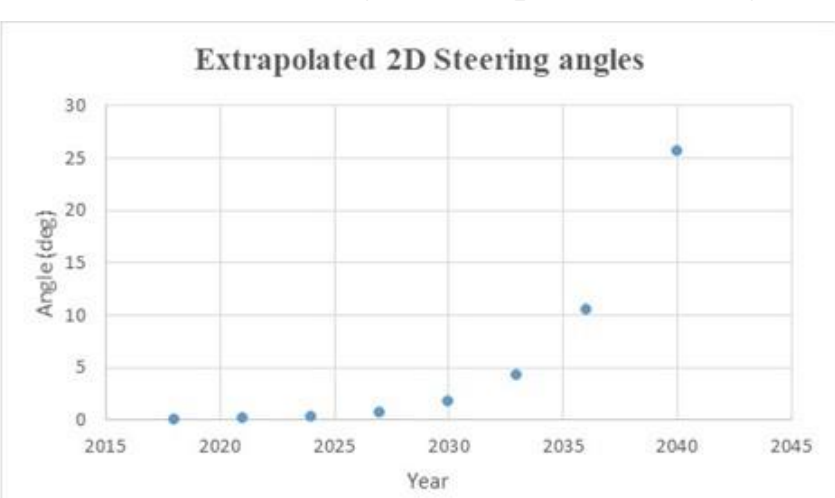

Figure 16: Extrapolated Chip scale steering angles for a $10 \mathrm{~cm}$ aperture 500 components, and the MOABB phase 1 program had 3000. We do this comparison for A $10 \mathrm{~cm}$ diameter aperture. This increase occurred over 3 years. At the same rate of increase by 2021 we would have 18000 elements. This is enough to do beam steering in a single dimension over plus or minus 15 degrees. The other possibility would be to use these elements in two dimensions, but combine chip scale beam steering with a large angle, step stare approach, such as polarization birefringent gratings, discussed below. While this is a dangerous extrapolation, because breakthroughs can come, it can provide a metric for how long it is likely to

\section{5: Pancharatnam based Non-Mechanical Beam Steering}

Non mechanical beam steering approaches discussed until now rely on creating OPD, which

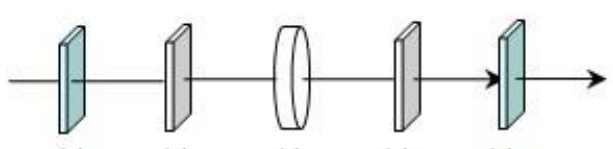

(a) (b)

Fig. 17: A basic setup of QHQ stack (a)\&(e):Polarizer; (b)\&(d):Quarter-wave Plate; (c): Half-wave plate is equivalent to a phase delay. There is an alternate approach which directly creates a phase delay. Figure 17 shows the optical model of the quarter wave plate, half wave plate, quarter wave plate, QHQ, device of Pancharatnam. A light beam passes through a polarizer, a quarterwave plate $(\lambda / 4$ plate $)$, a half-wave plate $(\lambda / 2$ plate), another quarter-wave plate, and another polarizer.

Linear incident light becomes circular polarized after the first $\lambda / 4$ plate, which can be defined as $E_{\text {in }}$ according to Jones Calculus notation:

$$
E_{\text {in }}=\left[\begin{array}{l}
E_{x i n} \\
E_{y i n}
\end{array}\right]=\left[\begin{array}{l}
E_{x i n} \\
i * E_{x i n}
\end{array}\right]
$$

or convenience, we assume it is a right hand circular polarized light. $E_{x i n}$, and $E_{y i n}$ are vector components along the $\mathrm{x}$ and $\mathrm{y}$ axis respectively.

The transmitted light $E_{\text {out }}$ is defined as a linear mapping of the incident light $E_{\text {in }}$ by a Jones Matrix which represents the $\lambda / 2$ plate: 


$$
E_{\text {out }}=\left[\begin{array}{cc}
\cos \beta & -\sin \beta \\
\sin \beta & \cos \beta
\end{array}\right]\left[\begin{array}{cc}
1 & 0 \\
0 & e^{i \varphi}
\end{array}\right]\left[\begin{array}{cc}
\cos \beta & \sin \beta \\
-\sin \beta & \cos \beta
\end{array}\right]\left[\begin{array}{c}
E x_{\text {in }} \\
i E x_{\text {in }}
\end{array}\right]
$$

Where $\beta$ represents the angle between the slow axis of the half wave plate and the $x$ axis, and $\varphi$ is denoted as the phase retardation of the half wave plate, which is equal to $\pi$. The final relationship can be simplified as:

$$
E_{\text {in }}=\left[\begin{array}{l}
E_{x i n} e^{i \bullet 2 \beta} \\
i * E_{x i n} e^{i \bullet 2 \beta}
\end{array}\right]
$$

In the last expression, the transmitted light is a left hand circular polarized light with a common phase factor $e^{i \cdot 2 \beta}$, which is the most important result from the simple model. The phase of the transmitted circular light can be accurately controlled by the azimuth angle, $\beta$. If $\beta$ varies horizontally from 0 to $\pi$ the spatial phase profile of transmitted light will vary horizontally from 0 to $2 \pi$. If we have a LC cell with an in-plane director, the azimuth angle linearly rotating from 0 to $\pi$, and the total OPD across the cell agreeing with the half-wave retardation for the design wavelength, then the final spatial phase profile of transmitted light will linearly change from 0 to $2 \pi$. By duplicating this spatial director configuration repeatedly, a LC grating without any fly back or reset can be created. The amazing thing is that the cell is only one half-wave OPD thick. The fly back, or reset, is eliminated in this device. The thin cell gap reduces light scatting and adsorption of the liquid crystal cell. With this type of device, even though the optical thickness is only $1 / 2$ wave, it is possible to create a constant large phase gradient over an aperture size only limited by manufacturing constraints.

\subsection{1: Polarization Birefringent Gratings}

Liquid Crystal polarization gratings, LCPGs, with nearly ideal diffraction efficiencies $(>99.5 \%)$ have been experimentally demonstrated over a wide range of grating periods, wavelengths (visible to near-IR), and areas (currently available up to $10 \mathrm{~cm}$ in diameter) ${ }^{31,32}$. Each polarization grating stage can double the maximum steered angle in one dimension without major efficiency reductions, so very large steered angles are possible (at least to $\pm 40^{\circ}$ field of regard). Like the birefringent prisms and saw tooth phase gratings it only requires a single stage of fine angle steering before the wide angle steering. This is in contrast to volume holographic wide angle steering that requires fine angle steering both before and after the holographic stack of glass. The structure at the heart of these devices is a polarization grating (PG), implemented using nematic liquid crystals (optionally switchable, or polymerizable). The nematic director is a continuous, in-plane, bend-splay pattern established using a UV polarization hologram exposing photo-alignment materials. When voltage is applied, the director orients out of plane, effectively erasing the grating. Diffraction occurs according to the following:

$$
\begin{aligned}
& \eta_{m=0}=\cos ^{2}\left(\frac{\pi \Delta n d}{\lambda}\right) \\
& \eta_{m= \pm 1}=\left[\frac{1 \mathrm{~m} S_{3}^{\prime}}{2}\right] \sin ^{2}\left(\frac{\pi \Delta n d}{\lambda}\right)
\end{aligned}
$$



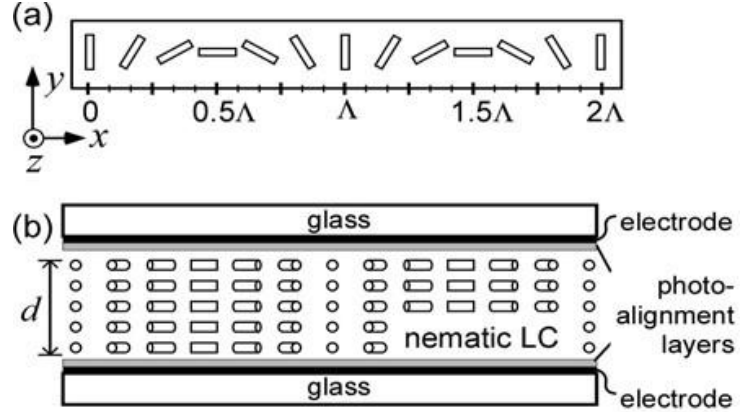

(c)

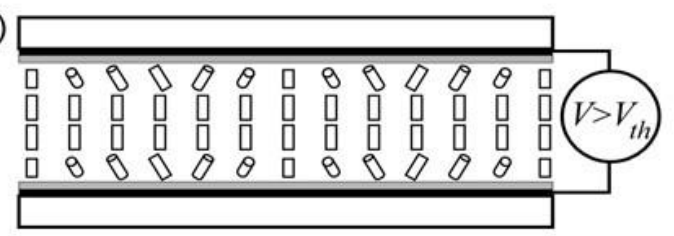

Fig. 18: The director profile of liquid crystal polarization gratings:

(a) top-view, and side-view in the zero (b) and high (c) voltage cases. where $\eta_{m}$ is the diffraction efficiency of the $m^{\text {th }}$ order, $\lambda$ is the wavelength of incident light, and $S_{3}^{\prime}=S_{3} / S_{0}$ is the normalized Stokes parameter corresponding to ellipticity of incident light. The grating equation applies. Note that only these three orders are possible, and that when the retardation of the LC layer is half wave $(\Delta n d=\lambda / 2)$, then $100 \%$ of the incident light can be directed out of the zeroth order. Note further that when the input polarization is circular, then all light can be directed into a single first order, with the handedness $\left(S_{3}^{\prime}= \pm 1\right)$ selecting the diffraction order (Figure 18). A single LCPG can be considered the key component within digital beam steerer with three possible directions $\left( \pm \theta\right.$ and $\left.0^{\circ}\right)$, as identified in Figure 18. For the non-diffracting case, an applied voltage reduces the effective birefringence toward zero $(\Delta n \rightarrow 0)$. LCPGs may also be fabricated with polymerizable liquid crystals,

also known as reactive mesogens, and would therefore be fixed indefinitely. The practical advantages of these passive PGs (over the switchable, or active, PGs) are that they tend to manifest less scattering losses and allow for smaller grating periods.

While Crawford and co-workers originally conceived the circular LCPG in its switchable and polymer forms, and demonstrated their basic diffraction behavior, their experimental results showed low efficiencies $(\leq 8 \%)$ and were limited to small diffraction angles. Subsequently, Escuti and co-workers developed materials and processing methods that produced defectfree, nearly ideal PGs with $>99.5 \%$ experimental diffraction efficiency (in the switchable and polymer variants) and grating periods from $\geq 5 \mu \mathrm{m}$ as a representative example. Around the same time Nelson Taberian was publishing somewhat similar work. ${ }^{33}$ NCSU however did receive a patent in the technical area. ${ }^{34}$

\subsection{2: VCOPA}

As discussed above LC optical phased arrays usually follow the idea of generating a linear change of optical path difference (OPD) across the aperture, while using resets to keep the required OPD small. Resets then impose a fly back region, reducing efficiency. As an alternative the V-COPA devices discussed here use the same basic physics as the QHQ device of Pancharatnam, discussed earlier. The difference is the steering angle of a single VCOPA device is variable instead of fixed like the previously discussed circular birefringent gratings. V-COPA devices also have approximately $99.5 \%$ diffractive steering efficiency, similar to birefringent prisms.

The LCPDs discussed earlier achieve their spiral structure through the use of an alignment layer that has the desired spiral structure. This approach obviously does not yield a tunable 
device. Kent State has demonstrated a variable device called the vertical - continuous optical phased array (V-COPA).

The basic structure of the device is shown in figure 19 by Lei Shi of Kent State. The alignment is approximately vertical at the top and bottom substrates. The alignment is quasivertical alignment because in some domains the alignment is not exactly vertical, but slightly tilts to the left, right or in and out of the plane of the paper. For example, the alignment over electrode \#1 and \#2, and over the gap between them, is slightly pointed into the plane of the paper. Similarly, the alignment over electrode \#3 is slightly pointed to the right, while the alignment over electrode \#6 is pointed to left, et al. All the other region's alignment keeps the initial vertical direction.

The cell is filled with $-\Delta \varepsilon$ LC materials, so when a voltage is applied to cause an E field that is vertical in the figure, the LC directors will distribute in the $\mathrm{x}-\mathrm{y}$ plane. The helical sense adopted is controlled by the slight tilting of the director alignment layer. The tipping of the director to the left and right in the figure 21 can be controlled by fringing fields resulting from the voltage pattern applied to the in-plane cell electrodes. In this way the regions tilting to the left or right can be controlled, and the sense of the helix can be electrically controlled.

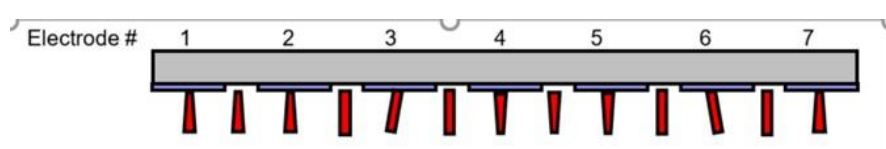

Side View

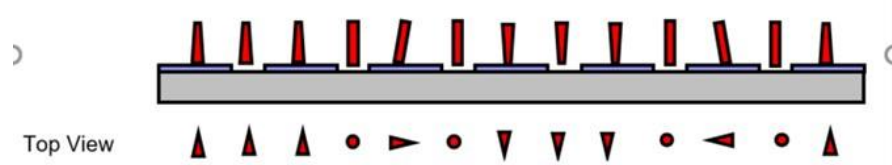

Fig 19: VCOPA alignment

By controlling the voltages applied to the electrodes the period of the helix can be changed. Tunable steering angle can be achieved. The main focus of current work on V-COPA is switching speed. If speed is sufficient then this will become a very attractive steering approach. It has high diffractive efficiency, like the birefringent prisms, but will require fewer layers. It is not clear that tunable devices will be manufacturable to steer to as large and angle as the fixed devices, so a future combination of VCOPA with a large angle step steering approach like birefringent prisms is likely.

\subsection{Non- Mechanical Steering of Broadband EO Beams.}

We started trying to steer broadband sensors in the late 80's to early 90's not long after we started the efforts to steer narrow band light non-mechanically. ${ }^{35,36,37,38,39}$ One of the early useful insights was that larger resets will be somewhat more broadband, even though it was not proven in a paper until 2005. Reference 40 shows in theory and experiment the effect of larger resets on the broadband nature of the beam steerer. ${ }^{40}$ While many avenues were pursued, the best approach for broadband beam steering was, and is, Achromatic Fourier transforms. The full width, half max, diffraction limit of aperture is

$$
\Delta \vartheta \approx \frac{\lambda}{D}
$$

Gratings steer to an angle proportional to wavelength for small angle steering. A longer

$$
\vartheta=\vartheta_{0} \frac{\lambda}{\lambda_{0}}
$$




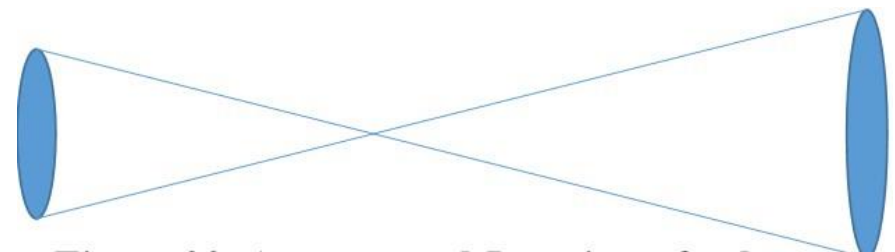

Figure 20: A conceptual Drawing of telescope

$$
\vartheta_{M}=\frac{\vartheta}{M}
$$

Where $\mathrm{M}$ is magnification. If we magnify by a factor of ten, then the steering angle is reduced by a factor if ten. Consider a telescope with magnification that is dispersive as follows:

$$
M=M_{0} \frac{\lambda}{\lambda_{0}}
$$

It has a basic magnification, but is dispersive, changing the steering angle. We can then get equation 6:

$$
\vartheta_{M}=\frac{\vartheta_{0} \lambda \lambda_{0}}{M_{0} \lambda_{0} \lambda}=\frac{\vartheta_{0}}{M_{0}}
$$

Note the wavelength dependence has disappeared.

But we need to develop a telescope with magnification given by;

$$
M=\frac{f_{1}}{f_{2}},
$$

Where $f_{1}$ and $f_{2}$ are the focal lengths of the two lenses in a simple telescope. We show a telescope in figure 3

To make a telescope that follows equation 23 we must make one of the focal lengths depend on wavelength. We could, for example, have

$$
f_{1}=f_{10} \frac{\lambda}{\lambda_{0}}
$$

If $\mathrm{f}_{1}$ changes then under normal circumstances lens \#1 no longer focuses at the same spot. This means the two lenses do not focus in the same spot, so the telescope becomes blurry, and is no longer is a useful telescope. We therefore need to have a "lens" with variable focal

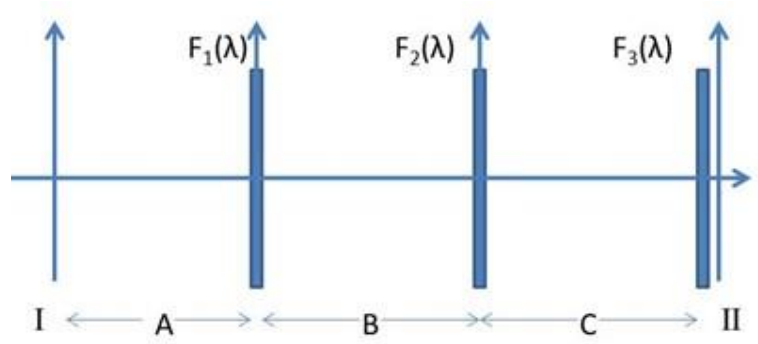

Figure 21: Lens Positions for an Achromatic Fourier Transform length, but a constant back focal distance. We want a lens that always focuses in the same spot, even though its focal length changes. An achromatic Fourier transform lens can have this property. The magnification amount could be just 1 to 1 as the basic magnification, with dispersion on top of that basic magnification. It could also be a different value if that would be useful in the design. Figure 21 shows a diagram of the 
internal make up of this achromatic Fourier transform (AFT) "lens"

We will only need the first two lenses. One of these lenses is diffractive. It has normal diffractive dispersion. The second lens has dispersion that is the opposite of lens 1 in the AFT, so the opposite of diffractive dispersion. The main challenge in designing the AFT is wavelength operation over a wide bandwidth.

In the 90's Dr McMnaamon had Dr Ed Watson hire Boulder Nonlinear Systems, BNS, and
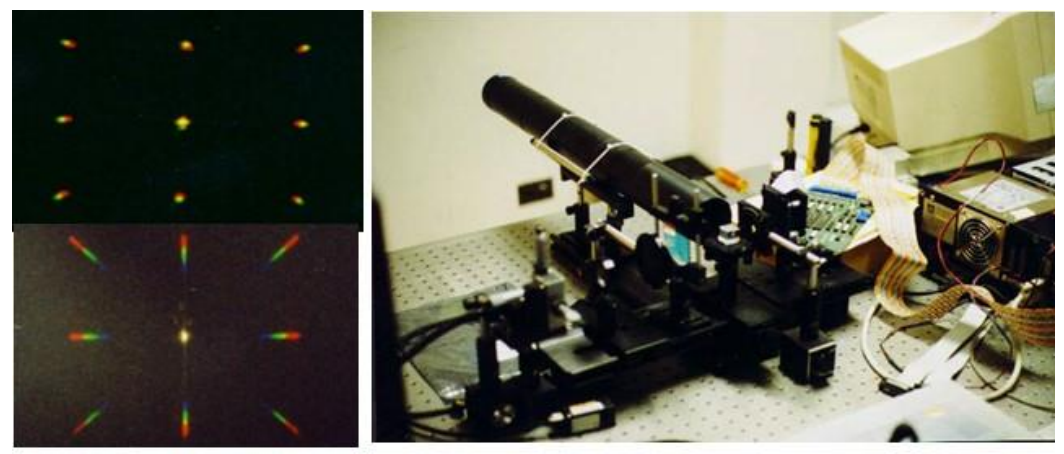

Figure 22: A broadband beam steering device Mike Morris's company to build a demo device the visible. It was for broadband beam steering. It is shown in figure 22 . The lens was longer then Dr McManamon wanted, but did work over a limited band in the visible. The actual device is shown on the right, and on the left we have the uncompensated steering of a point of broadband light on the bottom, and the compensated steering on the top. You can see the compensation is not perfect, but the colors are mostly brought back together. Magnification amount could be just 1 to 1 as the basic magnification, with dispersion on top of that basic magnification. It could also be a different value if that would be useful in the design.

\section{Summary:}

Non-mechanical beam steering has come a long way since the mid 80 's, when Dr McManamon first started working this technology. Initial work was mostly in liquid crystals. There was some acousto-optical work as well. Fringing fields limited the steering angle for LC OPAs. As a result larger angle step stare approaches were pursued. The first of those was volume holographic gratings, VHGs. While VHGs were successful the number of steering angles was linearly proportional to the number of volume holograms. More recently large angle step steering has been done using polarization birefringent gratings. VCOPA has been a very interesting method of steering too small to moderate angles. At this time work is occurring to speed up the steering time for VCOPA. EO crystals are becoming more promising, using higher EO coefficient crystals, and novel addressing structures. Chip scale OPAs are developing, but are still at an early stage. At this time they will be restricted to either one dimension, or small angle steering, by the number of addressable elements within the state of the art. Broadband non-mechanical beam steering in far behind narrow band nonmechanical beam steering, but is beginning to make progress.

\section{References:}

${ }^{1} \mathrm{~L} \mathrm{Wu}, \mathrm{SB}$ Maley, SR Dooley, TR Nelson..., A large-aperture, piston-tip-tilt micromirror for optical phase array applications- Micro Electro Mechanical Systems, 2008 
${ }^{2} \mathrm{~L}$ Wu, S Dooley, EA Watson, PF McManamon, A tip-tilt-piston micromirror array for optical phased array applications, , H Xie, Journal of Microelectromechanical Systems, 2010

${ }^{3}$ EA Watson, Analysis of beam steering with decentered microlens arrays - Optical Engineering, 1993

${ }^{4}$ Paul J. M. Suni(a), John Bowers(b), Larry Coldren(b), S.J. Ben Yoo(c), James Colosimo(a), “Coherent LidarOn-A-Chip", IEEE NAECON conference, 2017

${ }^{5}$ S. Pancharatnam, in Proceedings of the Indian Academy of Sciences, vol XLI, no. 4, sec. A, $137,1955$.

${ }^{6}$ P. F. McManamon, E. A. Watson, T. A. Dorschner, L. J. Barnes, "Nonmechancial beam steering for active and passive sensors", SPIE 1417, 110, 1991, p 194

${ }^{7}$ P.F. McManamon, J. Shi, and P. Bos, "Broadband optical phased-array beam steering", Opt. Eng. 44, 128004, 2005.

${ }^{8}$ J. Goodman, “ Introduction to Fourier Optics”, McGraw Hill, San Franciso, 1968, p 65

${ }^{9}$ J. Frank, J. D. Richards, "Phased Array Radar Antennas", in Radar Handbook, Merrill Skolnik, 3rd edition, New York, McGraw Hill, Ch 13, p13.2-13.3

${ }^{10}$ P.F. McManamon, “Agile Nonmechanical Beam Steering,” OPN, Mar, 2006 p 21 - 25.

${ }^{11}$ P. F. McManamon, T. A. Dorschner, D. C. Corkum, L. J. Friedman, D. S. Hobbs, M. K. O. Holz, S.

Liberman, H. Nguyen, D. P. Resler, R. C. Sharp, and E. A. Watson, “Optical Phased Array Technology," Proc. IEEE 84(2), 268-298, 1996.

${ }^{12}$ X. Wang, B. Wang, P. F. McManamon, J. J. Pouch, F. A. Miranda, J. E. Anderson, P. J. Bos, "Spatial resolution limitation of liquid crystal spatial light modulator", Liquid Crystal Conference, Great Lakes Photonics Symposium, Cleveland, OH June 7-11, 2004

${ }^{13}$ R. M. Matic, "Blazed phased liquid crystal beam steering,", Laser beam Propagation and Contr.: Proc. Soc. Photo Opt. Instrum. Eng., Vol 2120, pp 194- 205, 1994

${ }^{14}$ P.F. McManamon, Abtin Ataei., SYSTEM, METHOD, AND APPARATUS FOR ELECTRO-OPTICAL NON-MECHANICAL BEAM STEERING", filed Oct 27, 2017

15 https://en.wikipedia.org/wiki/PAVE_PAWS, downloaded Jan 3, 2019

16 Tomasz Piwonski, Jaroslaw Pulka, Evgeny A. Viktorov,1 Guillaume Huyet,1 and John Houlihan, Refractive index dynamics of quantum dot based waveguide electroabsorbers , APPLIED PHYSICS LETTERS 97, 051107 2010,

${ }^{17}$ Bekir C- akır a, YusufYakar b, AyhanO” zmen, Refractive index changes and absorption coefficients in a spherical quantum dot with parabolic potential, Journal of Luminescence132(2012)2659-2664

18 The company making these devices has been purchased by Analog devices, which has an interest in auto lidar applications. This will likely mean additional money will be invested in the engineering to bring this loss down, and to make these steering devices inexpensive. It is likely after the probably investment this will be an attractive beam steering technique when small apertures are applicable.

${ }^{19}$ T. Imai, M. Sasaura, K. Nakamura, and K. Fujiura, "Crystal Growth and Electro-optic Properties of KTa1-xNbxO3”, NTT Technical Review, NTT Photonics Laboratories Atsugi-shi, 243-0198 Japan. Email: tadayuki@aecl.ntt.co.jp

${ }^{20}$ H. Kogelnik, "Coupled wave theory for thick Hologram gratings", The Bell Systems technical Journal, Vol. 48, no 9, Nov. 1969, p 2909-2947.

${ }^{21}$ I. V. Ciapurin, L. B. Glebov, V. I. Smirnov, "Modeling of Gaussian beam diffraction on volume Bragg gratings in PTR glass", Practical Holography XIX: Materials and Applications, Proc. of SPIE Vol. 5742 (SPIE, Bellingham, WA, 2005) 0277-786X/05/\$15 · doi: 10.1117/12.591215

${ }^{22}$ L. Glebov, "Fluorinated silicate glass for conventional and holographic optical elements", Window and Dome Technologies and Materials X, Proc. of SPIE Vol. 6545, 654507, (2007)

${ }^{23}$ L. Glebov, "Volume Bragg Gratings in PTR Glass--New Optical Elements for Laser Design", 23rd Advanced Solid-State Photonics (ASSP) Topical Meeting. ASSP Technical Digest, Paper Code MD1, Nara, Japan.

${ }^{24}$ P.F. McManamon, “Agile Nonmechanical Beam Steering,” OPN, Mar, 2006 p $21-25$.

${ }^{25}$ Raytheon Steered Agile Beams, STAB, Final report, AFRL-SN-WP-TR-2004-1078; DTIC AD \# is : ADB 302140

${ }^{26}$ J. Sun, E. Timurdogan, A. Yaacobi, E. S. Hosseini1, \& M. R.Watts, "Large-scale nanophotonic phased array", January 10, 2013 | Vol. 493 |N AT U R E, p 19 5-199 
${ }^{27}$ D. Kwong, A. Hosseini, Y. Zhang, and R. T. Chen, “1x12 Unequally spaced waveguide array for actively tuned optical phased array on a silicon nanomembrane”, APPLIED PHYSICS LETTERS 99, 051104 (2011)

${ }^{28}$ J. Sun, E. Timurdogan, A. Yaacobi, E. S. Hosseini1, \& M. R.Watts, "Large-scale nanophotonic phased array", January 10, 2013 |Vol. 493 |N AT U R E, p 1 9 5-199.

${ }^{29}$ Paul J.M. Suni, James R. Colosimo S.J. Ben Yoo, John Bowers, Larry Coldren, Jonathan Klamkin, Photonic Integrated Circuit FMCW Lidar On A Chip, CLRC 2018, Japan. June 18-21

${ }^{30}$ Gordon Keeler, Laser Sensing and Communication Conference, OSA Imaging and Applied Optics Congress, Orlando, Florida, August 2018

${ }^{31}$ M. J. Escuti and W. M. Jones, "Polarization independent switching with high contrast from a liquid crystal polarization grating," SID Symposium Digest, vol. 37, pp. 1443-1446, 2006.

${ }^{32}$ M. J. Escuti and W. M. Jones, "A Polarization-Independent Liquid Crystal Spatial-Light-Modulator," Proc. SPIE - Optics \& Photonics Conference, vol. 6332, pp. 633222, 2006.

${ }^{33}$ Hakob Sarkissian, Svetlana V. Serak, Nelson V. Tabiryan, Leon B. Glebov, Vasile Rotar, and Boris Ya. Zeldovich, Polarization-controlled switching between diffraction orders in transverse-periodically aligned nematic liquid crystals, OPTICS LETTERS / Vol. 31, No. 15 / August 1, 2006

${ }^{34}$ https://patents.justia.com/inventor/michael-j-escuti, downloaded Jan 7, 2019

35 PF McManamon, EA Watson, Design of optical phased array beam steering with limited dispersion Aerospace Conference, 2001, IEEE Proceedings., 2001

${ }^{36}$ PF McManamon, EA Watson, Nonmechanical beam steering for passive sensors - Infrared Technology and Applications XXVII, 2001

${ }^{37}$ PF McManamon, EA Watson, TA Dorschner, LJ Barnes, Nonmechanical beam steering for active and passive sensors

- Infrared Imaging Systems: Design, Analysis, Modeling ..., 1993

${ }^{38}$ EA Watson, PF McManamon, LJ Barnes, AJ Carney, Application of dynamic gratings to broad spectral band beam steering - Laser beam propagation and control, 1994

${ }^{39}$ VG Dominic, AJ Carney, EA Watson, Measurement and modeling of the angular dispersion in liquid crystal broadband beam steering devices - Optical Engineering, 1996

${ }^{40}$ P. F. McManamon, J. Shi, P. J. Bos, "Broadband optical phased-array beam steering”, Optical Engineering 4412, 128004 December 2005

${ }^{41}$ G.M. Morris, Diffraction Theory for an achromatic Fourier transform", 1 June, 1981/ Vol 20, No 11/Applied Optics 